\title{
Concurrent Operation of Half- and Full-Duplex Terminals in Future Multi-Hop FDD Based Cellular Networks
}

\author{
Rainer Schoenen, Arif Otyakmaz and Bernhard H. Walke - ComNets, FB6, RWTH Aachen University, Germany
}

\begin{abstract}
Future OFDM-based cellular radio networks like IMT-Advanced systems are planned with both frequency division duplex and time division duplex in mind. Each of these duplex schemes has its benefits and drawbacks dependent on the scenario. In short-range communication and with small radio cells TDD is appropriate. For wide area cells FDD is preferred in general. If combined with multihop, i.e. the use of fixed relays, wide area cells can be built with a reduced number of base stations.

Economic rationale also leads to the requirements to produce cheaper terminal equipment. Full duplex FDD terminals can transmit and receive simultaneously, but need high quality, expensive RF duplex-filters, in order to separate uplink and downlink channels. Half-duplex FDD terminals are lower in cost and therefore an interesting solution. This paper shows how halfduplex terminals should be operated to achieve the same performance as full-duplex terminals. The coordination of half- and full-duplex terminal operation by the base station is a challenge. This paper introduces the resource scheduling algorithm located in the MAC layer and discusses implications and performance results especially for multihop cellular networks.
\end{abstract}

Index Terms-Half-duplex, full-duplex, FDD, Relaying, Multihop

\section{INTRODUCTION}

$\mathbf{N}$ EXT generation mobile radio networks of IMTAdvanced systems family will offer ubiquitous broadband high area coverage, at up to $1 \mathrm{GBit} / \mathrm{s}$ in cities and 100 $\mathrm{MBit} / \mathrm{s}$ in rural areas and quality of service (QoS) support in terms of throughput and low delay. Candidate technologies like 3GPP-LTE [1], WiMAX [2], as well as the Wireless World Initiative New Radio (WINNER) system design [3] are based on OFDMA transmission for flexible radio resource allocation, scalable and adaptable to both short range and wide area scenarios. Multihop relaying is part of these system concepts [3][6]. All technologies support Time Division Duplex (TDD) and Frequency Division Duplex (FDD) duplex transmission schemes, both having their pros and cons [7]. FDD requires operation of two distinct physical channels for transmitting downlink (DL) and uplink (UL) frames at the same time while TDD operates one channel only where DL and UL phases are interleaved in time. FDD is rather inefficient for handling asymmetric traffic. TDD is better for this case, because the DL/UL switching point can be chosen appropriately. Although it is quite static, too, because for interference mitigation DL and UL transmissions must be separated in space, time and frequency as much as possible. FDD does not need a guard time interval, so it is rather efficient in wide area scenarios with long signal propagation delay. Under high traffic load the base station (BS) may be able to take advantage of fullduplex FDD (FDFDD) operation, to full exploit its capacity, but a user terminal (UT) does not necessarily need to do so. Instead, half-duplex FDD (HDFDD) operation (either transmit or receive at any given moment) may be sufficient at a UT. FDFDD operation requires high quality, costly RF duplex filters to attenuate the received side-band power from its own transmission. In contrast, HDFDD UTs do not need such filters and therefore can be manufactured at much lower cost.

FDFDD and HDFDD are both applied in current mobile radio systems like GSM/GPRS, UMTS and WiMAX. GSM/GPRS applies HDFDD in general [8]. UMTS specifies FDFDD operation [9]. WiMAX also considers FDFDD operation [2], but TDD is used only. 3GPP-LTE favors FDD [1], and simultaneous use of HD and FD is aimed at, but so far details have not been worked out. Concurrent operation of HD and FD terminals in a multihop relaying capable system is desirable and not solved so far.

This work extends recent ideas [10] where the coexistence of FDFDD and HDFDD terminals in a radio cell was proven. There a memoryless radio resource scheduler has been used in the Medium Access Control (MAC) layer. By utilizing a more sophisticated scheduler with resource partitioning, the coexistence of FDFDD and HDFDD terminals in a radio cell is significantly improved. The radio frame organization is kept like common in OFDMA FDD systems [11], but for HDFDD two duplex groups (1 or 2) are introduced in order to alternate and interleave their DL and UL phases. The respective scheduling of resources enables the parallel operation of FDFDD and HDFDD terminals. It has been found that the limitations of HDFDD compared to FDFDD tend to reduce the fairness performance [10], especially in conditions of high traffic load, and the use of relays influences fairness even more. More precisely, the QoS-aware realtime traffic must be controlled below the saturation load, but the best effort or data traffic we treat here is expected to exceed the saturation point frequently, so there is definitely a need for fairness among user terminals. The observed unfairness in other solutions also meant that starting from a stable operating point, adding more and more terminals would lead into an unstable and unfair operating point. In this paper a solution to the fairness problem mentioned is proposed by providing a relay-aware proportional fair resource scheduler, together with a mid-term radio resource management responsible for partitioning resources to be used either at the first (hop 1 ) or the 
second hop (hop2) in a multihop scenario. Operating HDFDD only without FDFDD terminals is a special case of the above and works accordingly.

The paper is structured as follows. Section II defines the concept of HDFDD and FDFDD integration. In Section III scheduling of relay nodes (RN) is introduced. Scenarios and related simulation results are presented in Section IV. The paper ends with a concluding summary.

\section{SySTEM DESCRIPTION}

In an FDD system two frequency bands are used in parallel. The upper band is used for downlink (DL) transmission from BS to UT and from BS to RN and from RN to UT. The lower band is used for the uplink (UL), i.e. $U T \rightarrow B S, U T \rightarrow R N$, $R N \rightarrow B S$. BSs and RNs are assumed to operate in FD mode. UTs may be FDFDD capable or may only support HDFDD operation. Figure 1 shows the timing of FDFDD and HDFDD operation in an LTE superframe. The point with HDFDD operation is that the BS must know the actual transmission direction of a HDFDD UT, so that either DL or UL data is scheduled for a given time slot. The same applies for the relation between a RN and a UT. A change of the transmission direction within a time slot is not advisable [10].

The timing organization of the superframe and scheduling of the resources of each frame is performed by functional units of the MAC layer in the BS. It generates the frame timing signals and controls the resource partitioning (RP) and performs resource scheduling (RS) functions. Thereby it decides which resources in the frequency and time domain are to assign to a certain UT associated to the BS. With HDFDD UTs the resource scheduler allocates resources based on its knowledge whether a HDFDD terminal can be reached at a certain point in time or not. Fig. 2 shows the MAC superframe structure of the WINNER system for half-duplex operation [12]. Similar to LTE, see Figure 1, it comprises a preamble for synchronisation followed by eight frames each containing two so called chunks. Each frame starts with a resource map (RM) where the resource allocation information is broadcast to UTs associated to a BS. It contains the information which time and frequency slot has been reserved for which UT, to implement DL and UL transmission. If there are only HDFDD terminals around, there must be a way to time-interleave them so that some of them are in receive mode and some are in transmit mode. WINNER has proposed to introduce two half duplex groups, called 1 and 2. HDFDD UTs are partitioned into one of two groups and a UT stays in its group while it is associated to a BS. UTs belonging to group 1 receive in the first half of a frame and transmit in the second half whereas UTs of group 2 behave the other way around. Full-duplex terminals may transmit and receive and are scheduled in either part of the frame.

There is an obvious problem [10] with the proposal shown in Fig. 2: UTs of group 2 can not receive the resource map, since they transmit during the RM broadcast. Also, there is an unfairness concerning the resource distribution to the two groups, since group 1 gets less resources on DL than group 2. Without wasting resources it appears difficult to integrate

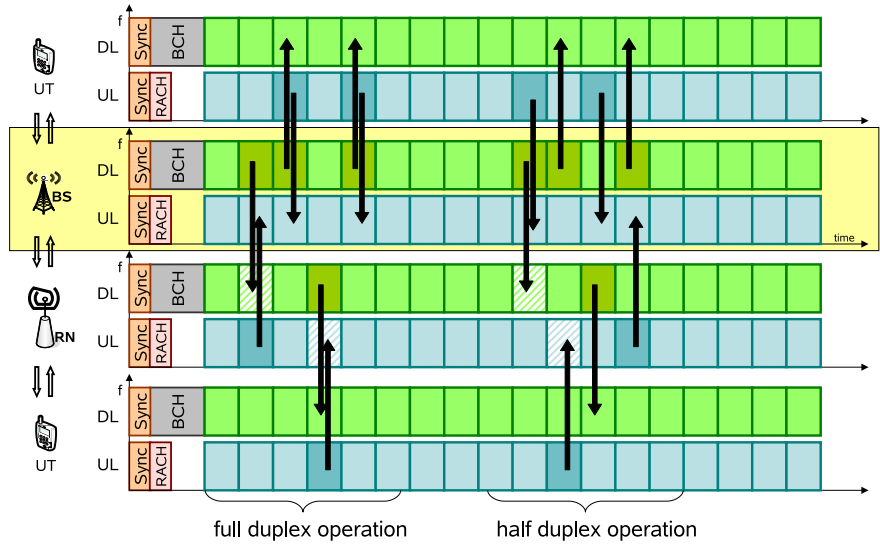

Fig. 1. FDD full and half duplex for 3GPP-LTE [11]

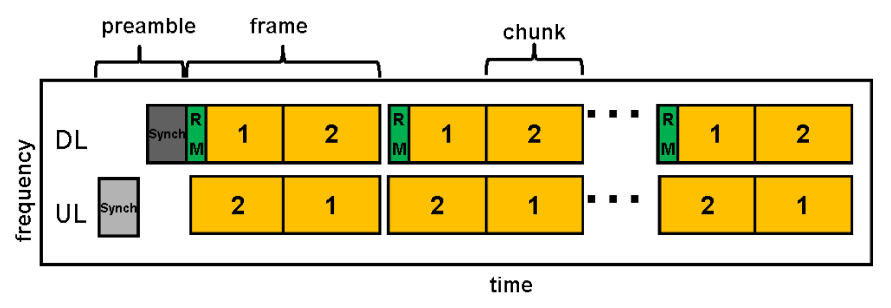

Fig. 2. WINNER MAC super-frame with two half-duplex groups [13]

RM signalling and fair handling of transmissions of both groups. From the proposals in [10] one solution appears to be most attractive, see Fig. 3. There the duplex groups alternate from frame to frame: In frame $2 i$ duplex group 1 receives the $\mathrm{RM}$ and in frame $2 i+1$ group 2 receives the RM. The information contained in the RM spans the contents of two chunks. According to Fig. 3 each group receives a RM every second frame, since both chunks in a frame are allocated to one group only. If both groups have the same number of UTs and the traffic is evenly shared by UTs, they are obviously treated fairly. FDFDD UTs must be scheduled so that they occupy the same amount of resources of both groups. When a map (scheduling result) is available every second frame it consequently is necessary to perform scheduling not only for one, but at least for two future frames.

\section{Multihop Scheduling}

Multihop systems feature at least one RN in the cell. RNs are useful to extend the coverage area of a radio cell or to increase the throughput capacity of a cell [4], [14].

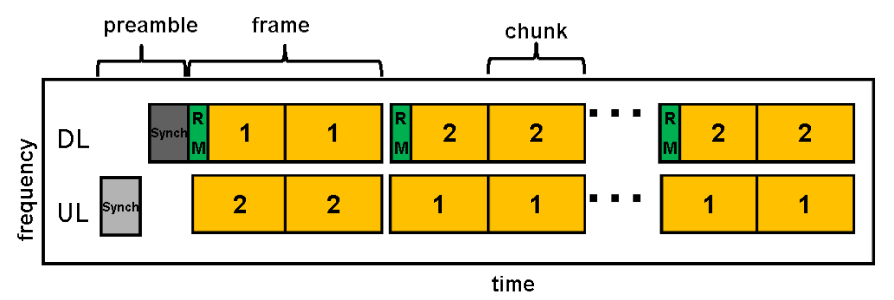

Fig. 3. Proposed MAC super-frame with alternating order of duplex groups 


\begin{tabular}{|l|l|c|c|c|c|c|c|c|c|}
\hline & BS & UT & BS & UT & BS & UT & BS & UT \\
\hline & DL \\
\hline
\end{tabular}

Fig. 4. Superframe with alternating TaskPhases, "BS" and "UT", of a RN

Relaying for FDD systems is preferentially organized in the time domain [11], i.e. radio resources (orthogonal OFDMA blocks in time and frequency domain) are partitioned among all serving stations (BS and RNs) and the roles alternate in time when a $\mathrm{RN}$ is in its serving role (actively responsible for resource allocation to its UTs) and in its UT role (passive, using resources allocated by the BS). Under this way of coordination no intra-cell interference is produced within a cell. Using spatial reuse among the resources controlled by RNs the spectral efficiency is raised [15] compared to where a time division multiplex (TDM) is assumed. The integration of half-duplex scheduling looks to be more complex at a first glance.

When the BS is owner of the resources, all UTs and RNs behave like UTs, i.e. a RN listens/receives on the DL channel and forwards the data on the UL channel to the BS. This is called the "UT" role of a RN. In the "BS" role the RN owns the resources which the BS has granted to it (by resource partitioning). In this role, the $\mathrm{RN}$ transmits on the DL channel and receives from it on the UL channel. A UT associated to a RN is called remote user terminal (RUT). This way of operation is compatible to the WINNER system concept [3], [16] where it is specified that RNs behave like a UT towards the BS and like a BS towards their UTs. The RN alternates its role from BS to UT frame by frame. Accordingly, two consecutive task phases of RNs can be identified that switch from frame to frame, namely BS and UT.

Fig. 4 depicts the super-frame structure of a RN. RNs like BSs operate in full-duplex mode. Therefore the FD link between RN and BS is scheduled without HD constraints. The BS must ensure only that the $\mathrm{RN}$ is in a task phase where it is acting as a UT when it is scheduled by the BS to receive the RM or DL data. During its "BS" role the RN schedules its associated RUTs belonging to one of two halfduplex groups. The RN scheduler must take into account the time intervals when it is in "BS" role and the time when a certain duplex group is reachable. Only when a RN is in the "BS" role and a specific HDFDD RUT is in receive phase, it can be reached on the DL. When a RN is in the "BS" role and the HDFDD RUT is in transmit phase, it may transmit data on the UL. A HDFDD RUT that is scheduled may no longer switch from frame to frame as described by Fig. 3 for a singlehop system, but it must toggle from BS phase to BS phase of its serving RN. Although drawn regularly in Fig. 4, in general the "BS/UT" task pattern of a RN need not be fixed. Therefore the resource scheduler in the RN must switch the UT group to be scheduled according to its task pattern. This is depicted in Fig. 5. The arrows shown originate from the responsible RM and the time when the scheduling decision was made. An arrow points to the frame scheduled to be served

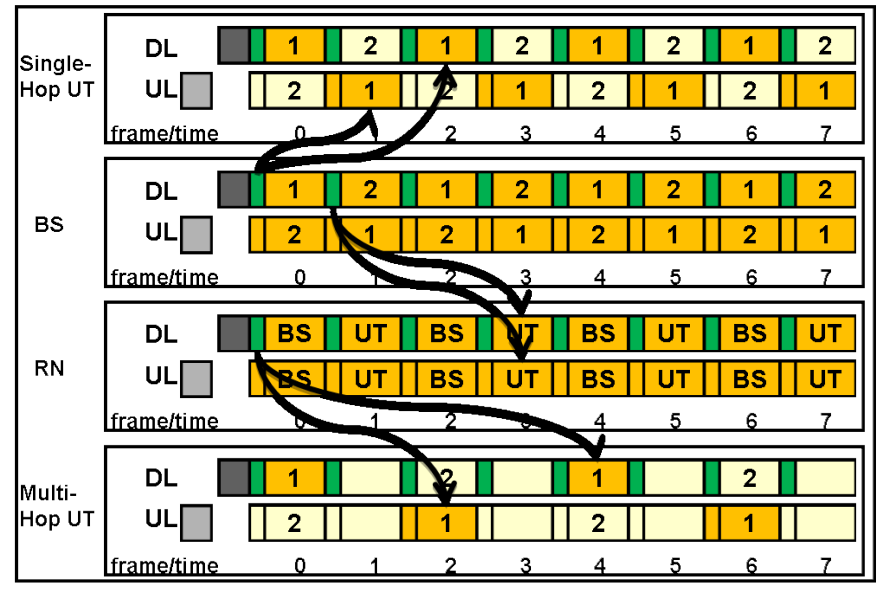

Fig. 5. Superframe for single- and multihop operation with HDFDD UTs and RN TaskPhases; The arrows show to which frame the RM points to; The numbers in the frames give the active duplex group

by the respective radio resource in the near future. By this it is ensured that the RM is received by HDFDD RUTs before the resources are really used in DL or UL. It can be seen in Fig. 5 that for singlehop UTs directly served by a BS the duplex group alternates from frame to frame as known from Fig. 3. But for RUTs served by a RN the duplex groups are toggled in multiples of the "BS" task phase of the RN. This is illustrated in the bottom row in Fig. 5. Obviously, there is a gap of one frame between the UL and DL phases of both duplex groups and a gap of three frames between consecutive DL and UL phases, respectively [10]. This is the reason for a higher delay experienced by RUTs, compared to singlehop UTs.

\section{Scenarios and Simulation Results}

In this section the half-duplex scheduling concept according to Fig. 5 is evaluated by event driven stochastivc simulation for some scenarios. The performance results are presented and discussed.

In general, six different classes of terminals are to distinguish, see Fig. 7. A scenario should at least comprise six terminals per cell, each representating a bunch of UTs of the same class. Otherwise, effects resulting from concurrent operation of these six classes cannot be studied. Using more RNs or more UTs of the same class does not provide more insight. The capacity available in a cell has to be shared equally among all six classes. Accordingly the scenario shown in Fig. 6 is used to study the class specific performances. It consists of one BS, one RN, three UTs associated to the BS and three RUTs associated to the RN. The UTs and RUTs are numbered. The lowest number is given to an FDHDD singlehop terminal, the second lowest number to a HDFDD singlehop terminal of group one etc.

First results in [10] showed that the duplex group scheduler works fine in the simulator. However, the UTs are treated very differently, class specific. To avoid unfairness the scheduler must a) actively ensure fairness over multiple frames and b) partition resources for hop 1 and hop 2 , so that the data rate required by each link can be met. 


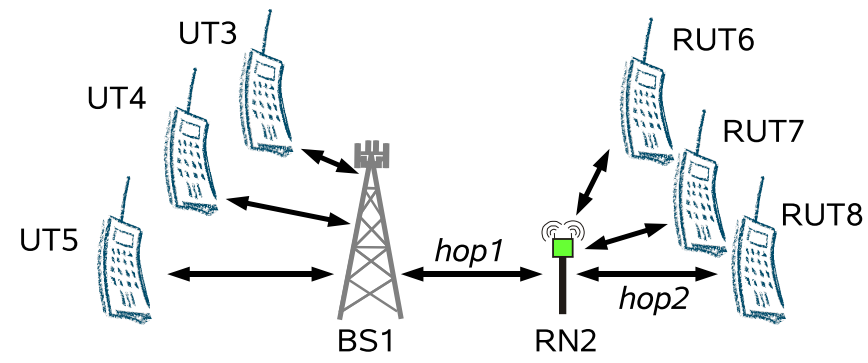

Fig. 6. Scenario with 1BS, 1RN, 3UTs and 3RUTs

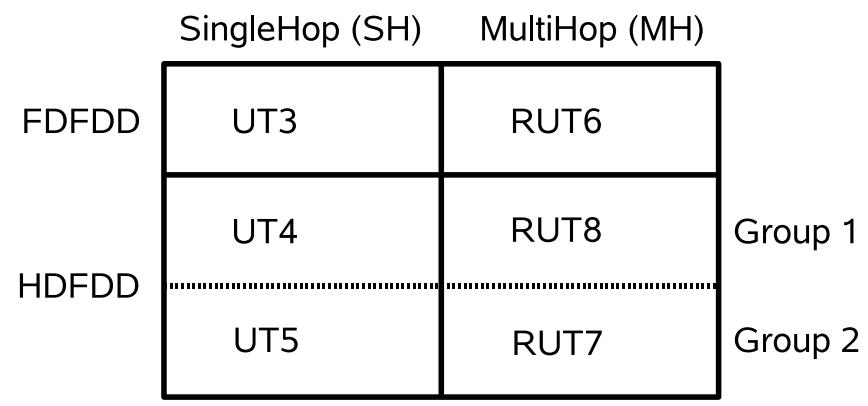

Fig. 7. UT classes according to duplex capability and group membership

Three scheduler are studied w.r.t. their performance:

- RoundRobin: memoryless,

- MaxThroughput: memoryless,

- ProportionalFair: stateful (scheduler with memory),

The RoundRobin and MaxThroughput schedulers are memoryless in the sense that they do not consider the past scheduling decisions or results for the current frame to schedule. The ProportionalFair scheduler keeps a state where the past data rate of each UT is stored and can be considered for future decisions [17], [18]. In fact the ProportionalFair scheduler is a closed loop control algorithm which aims at providing the same data rate $r$ to each active UT. It does so by preferring the connection with the lowest data rate in the past $\left(p_{i}\right)$, which is calculated from an exponentially weighted moving average per connection. For the moving average, a parameter $h=$ historyWeight is tunable in the state calculation, where $p_{i}$ represents the past data rate of a connection at time slot $i$, and $r_{i}$ the currently scheduled rate:

$$
p_{i}=h \cdot p_{i-1}+(1-h) \cdot r_{i}
$$

\section{A. Parameters}

The following parameters are fixed for all simulation studies. They are mainly taken from [12] and are listed in Tab. I. The distances between BS, RNs and UTs are chosen such that the highest PhyMode $Q A M 64-\frac{1}{2}$ can be used. The traffic model parameters are listed in Tab. II. To be able to operate RNs, the DL and UL bands are divided between the BS and the RNs during their BS task phase to avoid any intracell interference between these stations. This kind of resource partitioning is shown in Figure 8, it is kept simple and is therefore configured to be fixed for all simulation runs. As mentioned in Section III, the RN task phase switches from

\begin{tabular}{|l|l|}
\hline Parameter & Value \\
\hline superframe length & $5.8896 \mathrm{~ms}$ \\
frames per superframe & 8 \\
preamble duration & $0.36 \mathrm{~ms}$ \\
frame length & $0.6912 \mathrm{~ms}$ \\
OFDM symbol duration & $28.8 \mu \mathrm{s}$ \\
carrier frequency & $3.95 \mathrm{GHz} \mathrm{DL}, 3.7 \mathrm{GHz} \mathrm{UL}$ \\
channel bandwidth & $2 x 50 \mathrm{MHz}$ \\
number of subchannels & $1152(\mathrm{DL} \& \mathrm{UL})$ \\
OFDM symbols per frame for data & 21 in DL, 24 in UL \\
OFDM symbols for map & 3 \\
PhyMode (MCS) data & 64QAM1/2 \\
\hline
\end{tabular}

TABLE I

GENERAL SIMULATION PARAMETERS

\begin{tabular}{|l|l|}
\hline Parameter & Value \\
\hline Packet size distribution & synthetical typical IP traffic \\
mean packet size & $2056 \mathrm{bit}$ \\
packet interarrival time distribution & $\frac{2056 \mathrm{bit} \cdot \text { Number OfUTs }}{\text { TrafficRate }}$ \\
\hline
\end{tabular}

TABLE II

TRAFFIC MODEL PARAMETERS

frame to frame. Therefore only one of two consecutive frames is further subdivided in OFDMA frequency subchannels. The sections marked BS1 are the resources that belong to the BS while the sections marked RN2 are the ones assigned to the RN.

\section{B. Capacity Analysis}

For the parameter values shown in Tab. I the maximum achievable throughput $r_{t o t}$ can be calculated. According to [10] the theoretical maximum gross throughput for $\mathrm{UL}$ and DL is MaxThroughput ${ }_{U L}=112.6 \mathrm{Mbit} / \mathrm{s}$ and MaxThroughput $_{D L}=98.6 \mathrm{Mbit} / \mathrm{s}$, respectively. The overhead caused by the preambles and the RM are taken into account. The actual data rates are lower because of more overhead caused by CRC or unused resources due to packets which do not fully fit into the assigned resources. One single FDFDD UT alone would achieve the MaxThroughput values. A HDFDD UT can only achieve $50 \%$ of that. The same holds for a multi-hop full-duplex terminal, since it can also only be served every second frame due to the alternating task phases of its serving RN. A single RUT can therefore at most achieve $50 \%$ due to the resources needed in hop 1 .

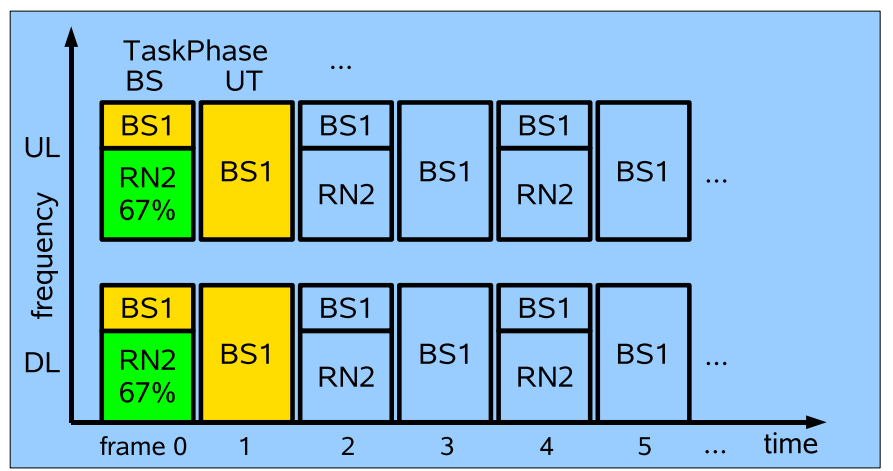

Fig. 8. Resource partitioning between BS and RN 
The lowest performance of $25 \%$ is expected for HDFDD RUTs. For simple scenarios and without special treatment, the performance of the different classes of UTs shown in Fig. 7 are proportional to the bounds given [10].

In this paper the scenario of Fig. 6 is studied where all classes of UTs are competing. The goal is to treat each UT equally fair, i.e. the data rate should be independent of the class membership in both UL and DL direction, always $\frac{1}{6}$, even under saturation. For RUTs the resources $R_{R U T, h o p 1}$ must be provided for the first hop of the traffic from/to the RUTs. They do not account for the goodput, instead they are considered overhead. Since the highest PhyMode is used on all links, $R_{R U T, h o p 1}$ requires the same amount of resources as $R_{R U T, h o p 2}$ on the second hop and $R_{U T}$ for the single hop UTs. Apparently, one third $(33 . \overline{3} \%)$ of the resources of a cell must be reserved for hop2, i.e. the resource partitioning share of RN2. Because of the two frame periodic switching of RN2 between "UT" and "BS" mode (Fig. 4) the reserved resources account for $66 . \overline{6} \%$ in the RN-BS frame and $0 \%$ in the RN-UT frame, which gives the nominal $33 . \overline{3} \%$. Fig. 8 shows the nominal resource assignment appropriate for this scenario. Following this way of partitioning, there are only $\frac{2}{3}=66 . \overline{6} \%$ of the resources left for goodput. In the end, each UT/RUT should get a share of $\frac{1}{6} \cdot \frac{2}{3}=\frac{1}{9}=11 . \overline{1} \%$ of the total maximum throughput $r_{t o t}$. In section IV-C we will find that these fair results can only be achieved with the ProportionalFair scheduler.

The calculations made for fair share partitioning and scheduling can be generalized to the use of arbitrary PhyModes: We define the "proportional fair share" in a way that any UT gets the same amount of resources $R_{i}$ on its last hop. If PhyModes are different, this amount of resources leads to a higher data rate $r_{i}$ for those UTs $i$ using a higher PhyMode. Let $M C S_{i}$ be the PhyMode on the last hop for $U T_{i}$ or $R U T_{i}$ and $M I_{i}$ be the mutual information in $[$ bits $/ \mathrm{s} / \mathrm{Hz}]$ for this PhyMode [19]. $M I_{R N}$ be the mutual information of hop 1 between $\mathrm{BS}$ and RN of a multihop connection. $M I_{\max }$ is $3 b i t / s / H z$ for $Q A M 64-1 / 2$. The amount of resources needed is $R_{i}=c \cdot \frac{r_{i}}{M I_{i}}$ with a constant $c$ that is cancelled out in the equations, later. The resources are limited as

$$
\begin{gathered}
R_{\text {tot }}=c \frac{r_{\text {tot }}}{M I_{\max }}=\sum R_{i}= \\
c \sum_{i=1}^{\# U T s} \frac{r_{U T_{i}}}{M I_{U T_{i}}}+c \sum_{i=1}^{\# R U T s} \frac{r_{R U T_{i}}}{M I_{R U T_{i}}}+c \frac{\sum_{i=1}^{\# R U T s} r_{R U T_{i}}}{M I_{R N}}
\end{gathered}
$$

Eq. (2) can be used to calculate the data rates of each UT and the required resources. If the fairness policy is to grant each UT the same amount of resources $R_{i}$ and to each RUT twice this number (to support hop 1 and hop 2 and a fair competition between UTs and RUTs) then we get using $c=\frac{R_{t o t} \cdot M I_{\max }}{r_{t o t}}$

$$
\begin{gathered}
R_{i}=\frac{R_{t o t}}{\# U T s+2 \cdot \# R U T s} \\
r_{i}=\frac{R_{i} \cdot M_{i}}{c}=\frac{r_{t o t}}{\# U T s+2 \cdot \# R U T s} \cdot \frac{M I_{i}}{M I_{\max }}
\end{gathered}
$$

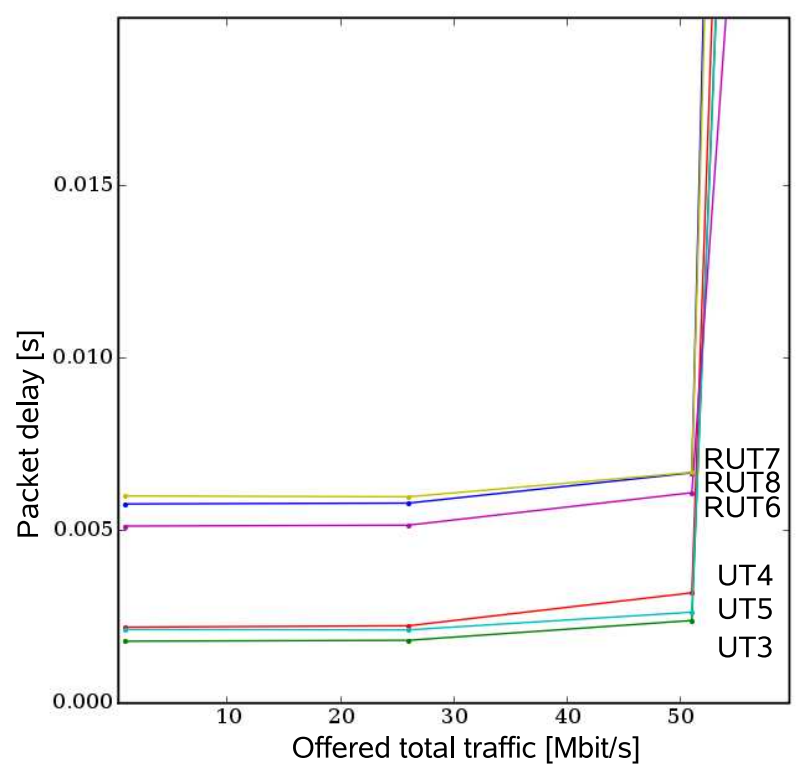

Fig. 9. DL Delay performance for Proportional Fair (stateful) scheduler. The $\mathrm{UL}$ is similar

If the goal is to provide each UT with the same rate $r_{i}=r$ then we end up with:

$r_{i}=\frac{r_{t o t} / M I_{\max }}{\sum_{i=1}^{\# U T s} M I_{U T_{i}}^{-1}+\sum_{i=1}^{\# R U T s} M I_{R U T_{i}}^{-1}+\sum_{i=1}^{\# R U T s} M I_{R N}^{-1}}$

Eq. (5) simplifies to $r_{i}=r_{\text {tot }} / 9$ with $M I_{x}=M I=$ const and $\# U T s=\# R U T s=3$. In general, Eq. (4) provides a higher total throughput compared to Eq. (5) if the PhyModes are different.

In principle, the difference in DL and UL data rate should be small. But the UL scheduling is based on less complete status information. The DL scheduler operates on the full local buffer state information. In the UL, the scheduler in the BS knows only the state sent to it during the uplink resource request. This information is delayed and may be outdated at the time it is used. However, due to sending the RM in the DL, the available DL resources are smaller than the UL ones, so that a somewhat smaller DL data rate can be expected.

\section{Results}

The (fair) resource partitioning is assumed as introduced in Fig. 8 and the task phases of the RN are chosen as shown in Fig. 4. In simulation campaigns the total offered load is increased from 0 to $180 \mathrm{Mbit} / \mathrm{s}$, well beyond the system capacity, so that the right part of the result graphs represents the overload condition. Up to the saturation point (of around $66 \mathrm{Mbit} / \mathrm{s}$ ) the performance of all UTs should be the same and in fact in all throughput result graphs (Fig. 10-13) we observe the expected linear increase with increased offered traffic. Below the saturation point, also the packet delay $d_{i}$ should be bounded (Fig. 9). This is not trivial, as one may think, because with an unsuitable RP and scheduler the performance 


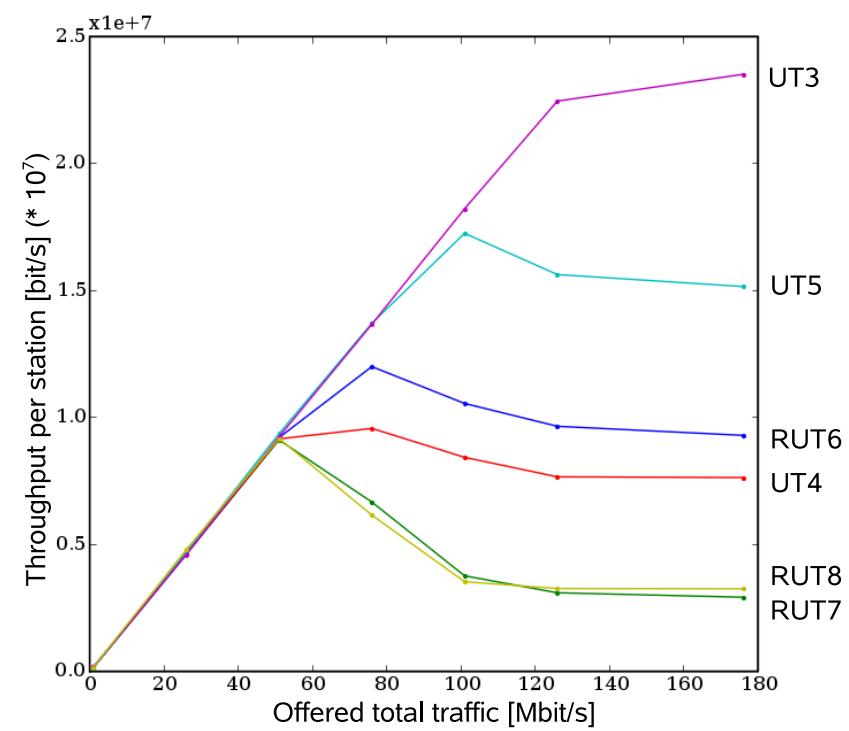

Fig. 10. DL Throughput for memoryless scheduler

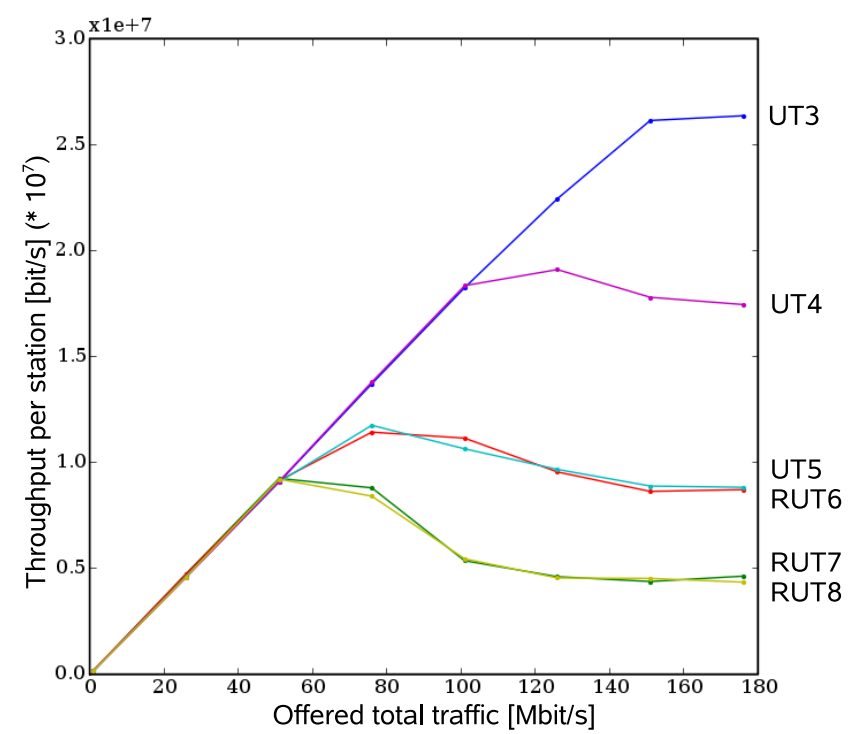

Fig. 11. UL Throughput for memoryless scheduler

also degrades below the saturation point [10]. The overload condition is also important, because with real traffic there are always overload phases of certain lengths.

The graphs show DL and UL throughput results for each of the six UT classes (one UT is representative for many UTs of the same class) and compare results of a memoryless scheduler (MaxThroughput, RoundRobin) and the (stateful) ProportionalFair scheduler, see Section IV. The memoryless scheduler provides fairness on frame basis, i.e. the scheduler takes only those stations into account which are currently reachable considering the duplex group.

The performance using the memoryless scheduler is shown in Fig. 10 for the DL and Fig. 11 for the UL. Under overload

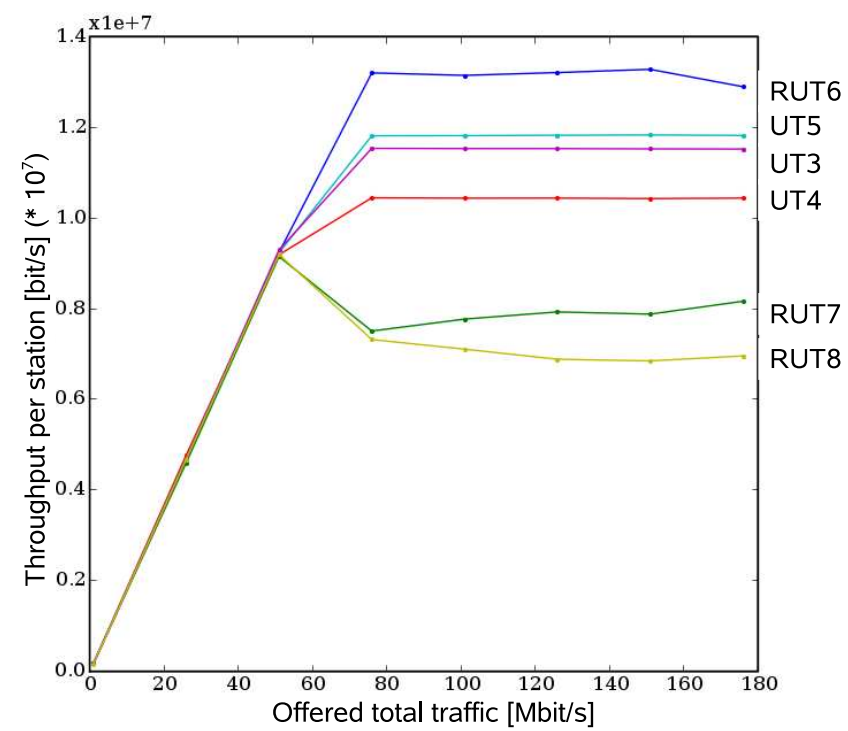

Fig. 12. DL Throughput for Proportional Fair (stateful) scheduler

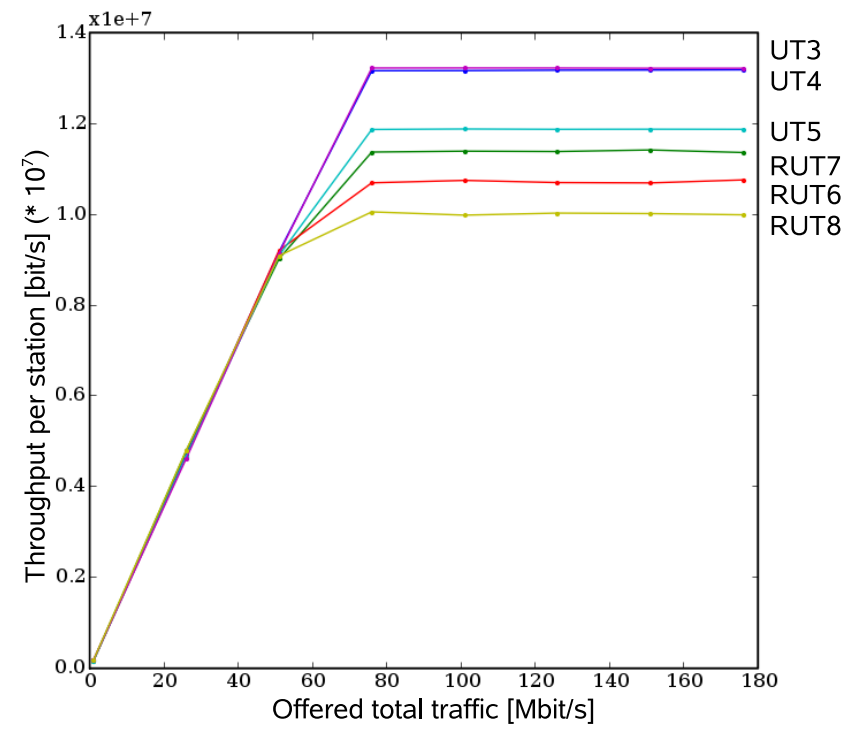

Fig. 13. UL Throughput for Proportional Fair (stateful) scheduler

we observe some interesting trends. UT3, as the FDFDD local terminal, has the advantage of being able to use all frames. Therefore it achieves the highest throughput of all UTs. In the DL, UT4 and UT5 differ significantly in throughput capacity, although they are both local HDFDD UTs. The reason is that UT5 (group 2) competes with RN2 during the RN's UT phase when the resource partitioning allows $100 \%$ of the resources. In the other frame UT4 (group 1) only competes with UT3 when only $33 \%$ of all resources are available due to the resource partitioning. In the UL the competition is vice versa, so UT4 performs better than UT5. Assuming that the first hop of the multihop connections is fair (aggregated flows), the big differences for the RUTs are that the FDFDD RUT6 can access 


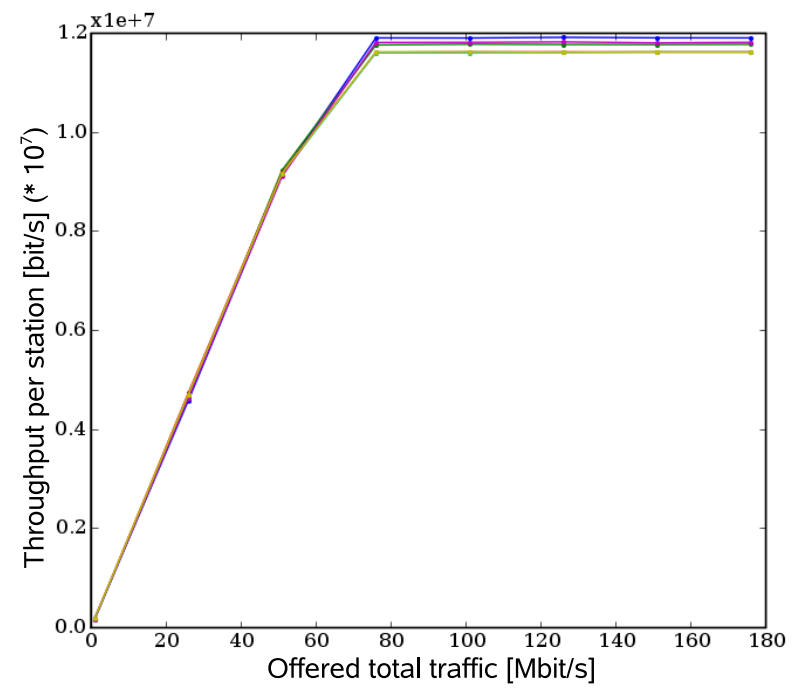

Fig. 14. UL Throughput for Proportional Fair (stateful) scheduler with historyWeight $=0.99$. This offers fair service to all terminals.

every frame where the RN is in the BS role. RUT7 and RUT8 can only compete for half of these resources, so they perform only at $50 \%$ of $r_{R U T 6}$.

The ProportionalFair scheduler remedies unfairness as shown in Fig. 12 and Fig. 13. Especially, in the UL all UTs and RUTs are treated quite the same below saturation ans similarly under overload. In the DL the UTs are also treated fair, despite their different duplex capabilities. The RUTs on average get the predicted $11 \%$ capacity share. The capacity distribution to the RUTs is not fair enough, but this seems to be a problem in the DL scheduler of RN2 in its BS role. By increasing the historyWeight parameter of the ProportionalFair scheduler from 0.9 to 0.99 , the result in Fig. 14 demonstrates the fair treatment of all terminals.

Fig. 9 shows the mean delay results of this scenario in the non saturated region. Under low traffic the average delay is almost constant, but clearly the singlehop UTs suffer only half the delay of the multihop RUTs. The saturation region is not shown, as it does not have a steady-state result.

\section{CONCLUSION}

This paper presents a concept for concurrent operation of half-duplex and full-duplex FDD user terminals in future cellular multihop mobile radio networks. The frame format and timing is controlled by a resource scheduler in shortterm, and resources are partitioned for the multihop links by quasi-static resource partitioning unit. Despite the limited capabilities of half-duplex FDD user terminals, it is shown that a well chosen resource scheduler and partitioning, together, can provide an equal and fair service to all terminal classes, almost independent of their duplex capability. The resource scheduler must consider the capabilities and actual availability of terminals for UL/DL transmission as well as the actual "role" of the relay stations. This study also reveils that a memoryless scheduler leads to unfair performance results.
The simulation based performance evaluation of half-duplex FDD terminals in single- and multi-hop scenarios showed that the duplex scheme integration using a ProportionalFair scheduler performs well and meets the results expected from mathematical analysis. The result is a system which performs as desired in both unsaturated and saturated traffic regions. Future research should investigate methods for an automatic mid-term resource partitioning strategy to adapt to changing load conditions and have QoS-aware scheduling goals that also meet other traffic contract requirements instead of fairness.

\section{REFERENCES}

[1] http://www.3gpp.org/Highlights/LTE/LTE.htm.

[2] http://www.wimaxforum.org/.

[3] http://www.ist-winner.org/.

[4] R. Pabst, B. Walke, D. C. Schultz, and et al, "Relay-Based Deployment Concepts for Wireless and Mobile Broadband Radio," IEEE Communications Magazine, pp. 80-89, Sep 2004.

[5] N. Esseling, R. Pabst, and B. Walke, "Delay and throughput analysis of a fixed relay concept for next generation wireless systems," in Proceedings of 11th European Wireless Conference 2005, vol. 1, Nikosia, Cyprus, Apr 2005, pp. 273-279. [Online]. Available: http://www.comnets.rwth-aachen.de

[6] IEEE, ”IEEE P802.16j/D1-2007, IEEE Draft Standard for Local and Metropolitan Area Networks, Part 16j: Air Interface for Fixed Broadband Wireless Access Systems - Multihop Relay Specification”, Aug 2007.

[7] Chan, P.W.C. et al, "The Evolution Path of 4G Networks: FDD or TDD?" IEEE Communications Magazine, pp. 42-50, Dec 2006.

[8] ETSI. GSM recommendations 04.05, 1993. Data Link Layer - General aspects.

[9] 3GPP, "TS 25 211, Physical Channels and Mapping of Transport Channels onto Physical Channels (FDD)",2001.

[10] A. Otyakmaz, R. Schoenen, and B. Walke, "Parallel Operation of Halfand Full-Duplex FDD in Future Multi-Hop Mobile Radio Networks," in European Wireless Conference, Prague, Jun 2008.

[11] R. Schoenen, R. Halfmann, and B. Walke, "An FDD Multihop Cellular Network for 3GPP-LTE," in Proceedings of the IEEE Vehicular Technology Conference (VTC'spring'08), Signapore, May 2008. [Online]. Available: http://www.comnets.rwth-aachen.de

[12] Winner II Deliverable D6.13.7, “Test Scenarios and Calibration Issue 2," http://www.ist-winner.org, Dec 2006.

[13] Winner II D6.13.14, "Final System Concept," http://www.ist-winner.org, Dec 2007.

[14] R. Schoenen, W. Zirwas, and B. Walke, "Raising Coverage and Capacity using Fixed Relays in a Realistic Scenario," in European Wireless Conference, Prague, Jun 2008.

[15] R. Schoenen, R. Halfmann, and B. Walke, "MAC performance of a 3GPP-LTE Multihop Cellular Network," in Proceedings of the IEEE ICC'08, Beijing, China, May 2008. [Online]. Available: http://www.comnets.rwth-aachen.de

[16] B. Walke, R. Pabst, L. Berlemann, and D. C. Schultz, "Architecture Proposal for the WINNER Radio Access Network and Protocol," in Proc. of Wireless World Research Forum (WWRF) 11th Meeting, Oslo, Norway, Jun 2004.

[17] A. Demers, S. Keshav, and S. Shenker, "Analysis and simulation of a fair queueing algorithm," in Proceedings of the ACM SIGCOMM, Austin, Texas, Sept. 1989, pp. 1-12.

[18] D. Stiliadis and A. Varma, "Efficient Fair Queueing Algorithms for Packet-Switched Networks," IEEE/ACM Transactions on Networking, vol. 6, no. 2, p. 175, Apr 1998.

[19] R. Schoenen and B. Walke, "On PHY and MAC performance of 3G-LTE in a multi-hop cellular environment," in Proceedings of the 3rd IEEE International Conference on Wireless Communications, Networking and Mobile Computing (WiCOM), Shanghai, China, Sep 2007. [Online]. Available: http://www.comnets.rwth-aachen.de 